\title{
Fast blood impedance measurements as quality indicators in the pre-analytical phase to prevent laboratory errors
}

\author{
Denise De Zanet ${ }^{1,2}$, Monica Battiston ${ }^{2}$, Elisabetta Lombardi ${ }^{2}$, Alessandro Da Ponte ${ }^{2}$, Ruben Specogna ${ }^{1}$, \\ Francesco Trevisan ${ }^{1}$, Antonio Affanni ${ }^{1}$, Mario Mazzucato ${ }^{2}$ \\ 1 Polytechnic Department of Engineering and Architecture, University of Udine, via delle Scienze 206, 33100 Udine (UD), Italy \\ 2 Department of Research and Advanced Tumors Diagnostics, CRO-IRCCS National Cancer Institute, via F. Gallini 2, 33081 Aviano (PN), Italy
}

\section{ABSTRACT}

In clinical laboratories, the major proportion of errors regarding blood analyses occurs in the pre-analytical phase. Pre-analytical conditions are key, necessary factors in maintaining the high quality of specimens, limiting day-to-day and batch variations, and guaranteeing the absolute reliability and accuracy of clinical results and related diagnoses. The quality of serum samples must be very high in order to avoid interferences due to hemolysis, thereby preventing measurement errors. In addition, the quality of the blood should always be fast monitored to identify inadequacies and guarantee their complete usability in transfusion procedures. In the near future, the solution could be to supply laboratories with smart and portable devices that are able to perform fast quality tests for every sample. Electrical impedance has relevant potential in analyzing and monitoring blood quality. We propose a new, simple impedancebased biosensor that can perform accurate and efficient single and multi-frequency impedance measurements in the pre-analytical phase and to check the quality of blood samples using quantitative thresholds as useful indicators to ensure the reliability of results and thereby prevent laboratory errors. The proposed sensor allows for discriminating different blood components, identifying hemolysis in serum, evaluating blood quality, and rapidly quantifying its hematocrit.

\section{Section: RESEARCH PAPER}

Keywords: electrical impedance; measurements; blood components; laboratory errors; pre-analytical phase management

Citation: Denise De Zanet, Monica Battiston, Elisabetta Lombardi, Alessandro Da Ponte, Ruben Specogna, Francesco Trevisan, Antonio Affanni, Mario Mazzucato, Fast blood impedance measurements as quality indicators in the pre-analytical phase to prevent laboratory errors, Acta IMEKO, vol. 7, no. 4, article 6, December 2018, identifier: IMEKO-ACTA-07 (2018)-04-06

Editor: Alexandru Salceanu, "Gheorghe Asachi" Technical University of lasi, Romania

Received March 28, 2018; In final form May 31, 2018; Published December 2018

Copyright: (C) 2018 IMEKO. This is an open-access article distributed under the terms of the Creative Commons Attribution 3.0 License, which permits unrestricted use, distribution, and reproduction in any medium, provided the original author and source are credited

Corresponding author: Denise De Zanet, e-mail: dezanet@cro.it

\section{INTRODUCTION}

Blood is a biological fluid that circulates throughout the body and contains extensive information on the state of a person's health and disease. It is composed of a conductive liquid (plasma) and a suspension of particles (red blood cells, white blood cells, and platelets). Once blood coagulates, cells aggregate to form red clots, and the surrounding liquid becomes serum. When making decisions about diagnoses, necessary further investigations, or patient management, clinicians rely on the results of blood laboratory analyses. It is, thus, clearly necessary to ensure the reliability and the accuracy of these analyses in healthcare to guarantee that the laboratory tests accurately reflect the patient's health state.

A huge number of control systems have been developed and used in most clinical laboratories to maintain high quality in the analytical phase [1]. This is not the case for the pre-analytical phase. The majority of laboratory errors are made in this phase, which covers all processes from the time that a laboratory test request is made by a physician until the sample is ready for testing. The magnitude of the effect of these errors on patient care is significant, and the focus of healthcare institutions is the improvement of laboratory test quality, renewing the interest in pre-analytical processes. Thus, the use of quality controls to prevent laboratory errors should be an essential activity for any healthcare laboratory [2]-[5]. Unfortunately, despite that several methods and systems are currently used in clinics for the preparation of quality control samples [6], [7], in practice, they depend on high dimensions instrumentation and specific commercial kits, whose usage procedures require optimization.

The most frequent reason for the inadequacy of a sample, such sample being able to cause analytical errors in laboratories, is represented by hemolysis (i.e., the rupture of red blood cells), giving rise to the release of intracellular content, such as 
hemoglobin. Free hemoglobin causes interferences in many dosage methods and colorimetric analysis and makes serum samples unsuitable for analysis. Another inadequacy in a sample is that the serum contains minute white clots, i.e., clots without red cells that are made by a structural net (called fibrin) that forms during coagulation. Limited presence of hemolysis or white clots is a sufficient basis for technical problems and measurement disruption [8], [9]. Nevertheless, the quality of serum in the pre-analytical phase is usually measured only for samples that visibly show pale red hemolytic shades, while it should be rapidly and always tested for every sample, without exclusion, in order to assure the complete accuracy of laboratory analyses.

In addition, quality control of every sample before laboratory analysis or transfusion is not always provided for blood that is collected from both healthy donors and patients. Consequently, the identification of inadequacies and the prevention of measurement errors are not guaranteed. Anomalies involving the temperature of the containers for the transport and storage of blood are frequent and common and can lead to variations in blood consistency and to its undesired coagulation. These problems should be identified and monitored by means of auxiliary investigations to adequately preserve the samples and to rapidly identify the opportune corrective actions. Accordingly, changes in hematocrit value, i.e., the volume of red blood cells as a percentage of the whole blood volume, could represent a useful indicator of transport and storage conditions quality. Thus, the quality of blood samples should be always quantified quickly to guarantee their complete compatibility and efficiency in transfusion procedures and to avoid anomalies and errors in laboratory analyses.

Electrical measurements represent a highly promising tool for analyzing blood. The relevance of the impedance measurement technique for characterizing biomedical human aspects has already been proposed in [10], [11]. Blood consists of conductive plasma and suspended cells (especially red blood cells, but also white cells and platelets). Cells are composed of an outer lipidic capacitive membrane, which contains an intracellular fluid with dielectric and resistive properties that are similar to plasma. Damage to the membrane and, consequently, to its functions is lethal for the cell. Ordinary circuit elements, such as resistors and capacitors, are considered as lumped-constant quantities, which have ideal properties. With the aim of modeling blood in terms of its components and dynamic behavior, ideal circuit elements may be inadequate in describing its electrical response. In addition, the interaction of blood with electrodes is too complex to be represented with the ideal response expected for a single electrochemical reaction. The interaction between a biological fluid and a metal electrode forms a narrow interface, known as a double layer, where the current flows as in a capacitive layer, but the current phase shift, in magnitude, is less than $-90^{\circ}$. Both the blood and the double layer are thus modeled using the Constant Phase Element (CPE) [12] in the equivalent circuit. The CPE is considered a distributed impedance element, examining the imperfect responses of real systems whose impedance is defined as $\dot{Z}_{C P E}(\omega)=1 /\left[C_{C P E}(j \omega)^{\psi}\right]$ [12]. The parameter $\psi$, with $0 \leq \psi \leq$ 1 , is the phase shift or phase angle, while $\mathrm{C}_{\mathrm{CPE}}$ is the capacitance. When $\psi=0$ the CPE resembles a pure resistor, instead, when $\psi=1$, the CPE looks like an ideal capacitor.

Measurements for the characterization of whole blood dynamic behavior in artificial micro-channels have been presented using electrical impedance spectroscopy, as described in [13]-[17], by using an impedance meter and a low-cost sensor based on printed circuit board technology. With regard to the characterization of single blood components, this challenge is still open and has not yet been settled by a single multimeasurement sensor.

Here, we propose a new, simple, impedance-based sensing device. Comparing and combining the data on impedance measurements, we are able to characterize and discriminate different blood components and to perform fast and accurate controls to test the quality of blood and serum samples, with the possibility of confirming or rejecting their usability in clinics.

\section{MATERIALS AND METHODS}

For each experiment, $3 \mathrm{ml}$ of venous blood was collected into citrate as anticoagulant (hematocrit of $38 \pm 3 \%$ ) from each of the healthy blood donors who participated in this study. Informed written consent was obtained from the participants according to the Declaration of Helsinki and the DMS of the Italian Ministry of Health on November $2^{\text {nd }} 2015$ (concerning the quality and safety of blood and blood donors). The Ethics Committee CRO-IRCCS Aviano approved the study. Afterwards, each vial of whole blood was divided into three parts with the aim of obtaining the materials demonstrated in Figure 1a. One part of the whole blood $(1 \mathrm{ml})$ was left untreated (whole blood shown in Figure 1a). Another part of the whole blood $(1 \mathrm{ml})$ was centrifuged to obtain, as shown in Figure 1b, plasma (a liquid substance with all the coagulation proteins, but without cells) and whole concentrated blood (red cells, white cells, and platelets, with a hematocrit of $60 \pm 3 \%$ ). Finally, for the last $1 \mathrm{ml}$, $\mathrm{CaCl}_{2}$ (5 $\mathrm{mM}$ final concentration) was added to stimulate the coagulation process and to obtain serum (a liquid substance without cells and without coagulation proteins) and the red clot (red cells, white cells, platelets, and fibrin). The serum thus extracted can appear yellow (the serum in Figure 1a) or it can show pale red shades (the serum with low or high hemolysis in Figure 1a, c, and d), a visible indication of the presence of hemolysis due to damage to red blood cells. In addition, serum can sporadically contain fibrin white clots. If the serum expresses hemolysis, the white clot can contain fragments of damaged red blood cells, and it looks light red (the white clot with hemolysis in Figure 1a). These anomalous situations, not always identifiable by eye, can lead to technical problems and measurement interferences if they are not rapidly recognized.

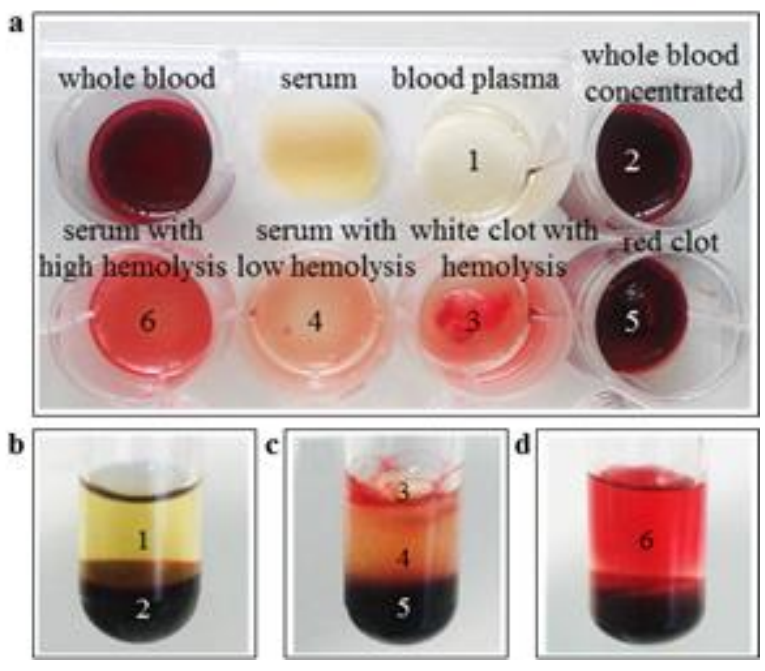

Figure 1. Blood components characterized with impedance measurements. Components disposed in plastic wells (a). Whole blood centrifuged (b). Blood coagulated and centrifuged $(c, d)$. 

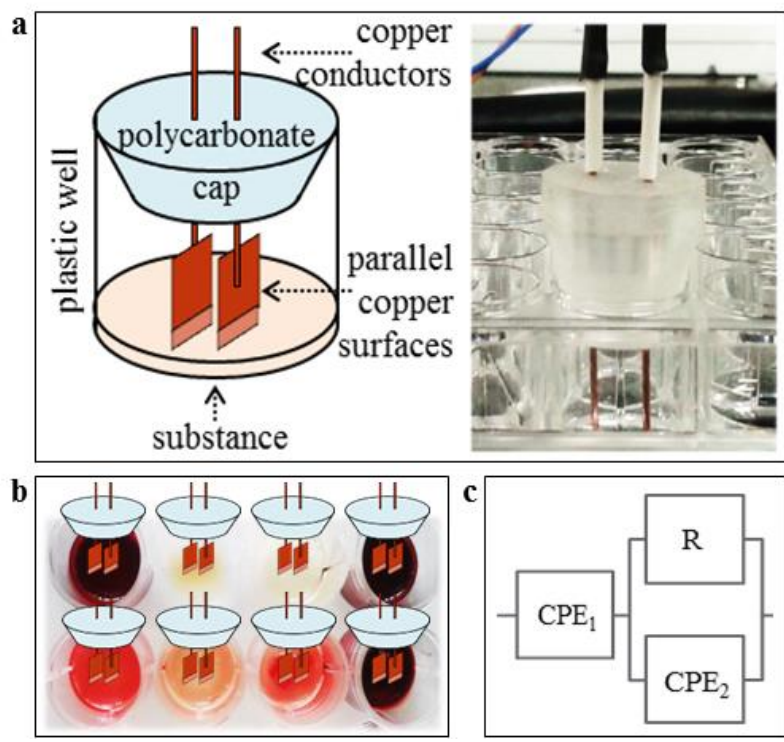

Figure 2. Sensing module for electrical impedance measurements. A polycarbonate cap is placed on each well, and the parallel copper conductive surfaces are immersed in the fluid substance $(a, b)$. Equivalent circuit (c)

Each blood component was homogeneously disposed in a single well ( $200 \mu \mathrm{l}$ of substance, well diameter: $16 \mathrm{~mm})$ and their electrical impedance was measured by using a low-cost, small, and reproducible sensing module with a simple geometry (Figure 2a). The sensing module appears as a capacitor with parallel planar surfaces made of copper (width: $7 \mathrm{~mm}$, distance between surfaces: $4 \mathrm{~mm}$ ). A polycarbonate cap supports the accurate positioning of the capacitor in the blood substance (as schematically shown Figure $2 \mathrm{a}$ and Figure $2 \mathrm{~b}$ ) and, consequently, maintains the stability of the measurements. The device was connected to a high-precision LCR meter [18] in order to perform the impedance measurement in the frequency range [100 Hz, $2 \mathrm{MHz}$ ] with 20 logarithmic spaced steps. A drive voltage of $50 \mathrm{mV}$ was chosen, considering the electrochemical properties of the materials, and open-short calibration was applied with the aim of reducing parasitic effects.

The equivalent lumped circuit is presented in Figure 2c. It allowed for a satisfactory correlation between the experimental data and the physical processes that govern the system. The double layer at the interface is represented by $\mathrm{CPE}_{1}$, characterized by capacitance $C_{1}$ and phase shift $\psi_{1}$, whose impedance is $\dot{Z}_{\mathrm{CPE} 1}(\omega)=1 /\left[C_{1}(\mathrm{j} \omega)^{\psi / 1}\right]$. Furthermore, according to the purely resistive behavior of the extracellular fluid and considering the necessity of representing different cellular and membrane conditions with a generalized and versatile circuit element, blood was modeled in terms of a parallel between the resistance and the CPE. The $\mathrm{CPE}_{2}$ models the cells with their membrane and is characterized by capacitance $C_{2}$ and phase shift $\psi_{2}$, and exhibits and impedance $\dot{Z}_{\mathrm{CPE} 2}(\omega)=1 /\left[C_{2}(\mathrm{j} \omega)^{\psi_{2}}\right]$, while resistance $R$ represents the fluid in which the cells are suspended.

The blood of $n=20$ different donors, equally distributed in terms of gender, was collected and analyzed, obtaining impedance signals varying with frequency and stable in time. In addition, the hematocrit was measured for all the blood samples with a commercial instrument that is commonly used in clinical laboratories.

\section{RESULTS AND DISCUSSIONS}

\subsection{Characterization of blood components}

Impedance signals related to the different blood components of Figure 1 are displayed in Figure 3 as magnitude (left panel) and phase (right panel). For each material, the average signal, with its related standard deviation, is illustrated $(n=20)$. It is evidently possible to use this new prototype to discriminate blood components on the basis of their impedance magnitude and phase, which are representative of their resistance to the current flow and depend on the amount of the capacitive contribution due to the lipidic membrane (mostly of the red blood cells). In particular, observing the magnitude of impedance at the central frequencies in Figure 3, from the bottom to the top, following a decrease of conductivity, we can observe in order: white clot, plasma, serum, white clot with hemolysis, serum with increasing levels of hemolysis, whole blood at different densities, and red clot. The properties of blood components, revealing that the electrical conductance decreases as the red blood cells concentration increases, can also be qualitatively appreciated from the phase diagrams of Figure 3, where it is evident that all the blood components without red cells or containing damaged

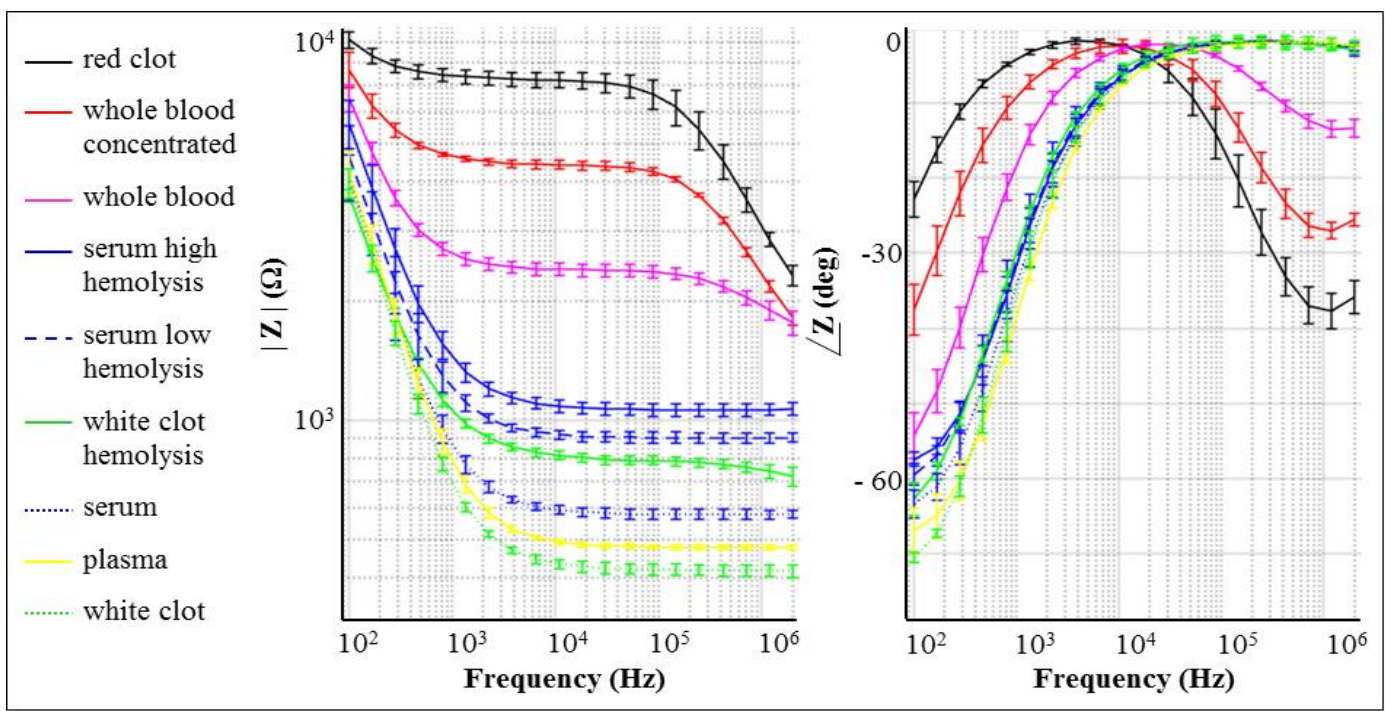

Figure 3. Electrical impedance signals displayed as magnitude (left panel) and phase (right panel). Each blood component is represented in terms of mean and standard deviation $(n=20)$. 
red cells have an impedance phase that is distinguishable from the impedance phase of samples with healthy red blood cells.

In addition to the evident capability of characterizing different blood components with electrical impedance measurements, the most promising results (presented in Figure 3) are shown by the curves of serum (pure or with hemolysis as well as those relative to the blood), specifically the combination that comprises whole blood, whole concentrated blood, and red clot. These results allow for associating and proposing a specific quantitative preanalytical threshold value for the rapid quality control of serum and whole blood samples. According to the impedance magnitude signals analyzed and shown here, we can thus confirm that serum and whole blood samples can be accepted for laboratory analyses and transfusions if their impedance magnitude levels at the central frequencies measured with our sensor do not exceed $700 \Omega$ and $3 \mathrm{k} \Omega$, respectively.

An interesting point that deserves attention and further experiments is represented by the white clot curves. In Figure 3, white clots without hemolysis seem to be the most conductive material among those analyzed, which is because the fiber net is a highly conductive structure of fibrin, and there are no resistive cellular components inside. By using this approach, fibrin could be electrically described, and its formation could be identified and monitored in real time during the whole physiological process of coagulation.

The discrimination of blood components through the impedance signals was confirmed and enriched by the inverse analysis performed using complex non-linear least square (CNLS) fitting software. Thanks to this software, it was possible to quantify each single lumped elements parameter introduced in Figure 2c $\left(C_{1}, \psi_{1}, R, C_{2}, \psi_{2}\right)$ with an uncertainty in the order of $2 \%$. The numerical parameters estimated, related to the equivalent circuit of Figure 2c, are displayed in Table 1 and are expressed in terms of mean and standard deviation $(n=20)$. An important aspect of the electrical characterization of a system is the possibility to identify and associate equivalent circuit elements with specific physical processes occurring. In particular, the parameters of $C P E_{1}$, related to the double layer, $\left(C_{1}, \psi_{1}\right)$ appear to be almost equal among all the blood components analyzed. In fact, the double layer is common among all the blood components because it is associated with the interface interaction between the material and the metallic electrodes. For this reason, $C P E_{1}$, representing a common electrical behavior, can be compensated with the aim of evidencing the specific electrical properties of each biological material, dealing with the specific electrical response of the material alone. The numerical results confirm that the $C P E_{2}$ is directly related to the presence or the absence of cells (mostly red blood cells) and the possible breaking of their membrane. In particular, the values of the $C P E_{2}$ parameters $\left(C_{2}, \psi_{2}\right)$ and $R$ are significantly different if there are cells (the higher the number or concentration, the higher the value), if cells are absent, or if there are cells with a damaged membrane (the hemolysis condition). In addition, the phase shift of $\mathrm{CPE}_{2}, \psi_{2}$, measures exactly one when cells are absent or their membrane is damaged (blood plasma and serum), indicating that, in such cases, $C P E_{2}$ behaves like an ideal capacitor, with a very low capacitance $C_{2}$.

Interpolations of frequency points are shown in Figure 4, for all the blood components analyzed, in a real-imaginary plot, without double-layer compensation (the left panel) and with compensation (the right panel), which highlights the differences between the samples on the basis of their intrinsic characteristics. Imagining the natural extension of the compensated curves in Figure 4 towards infinite frequencies, it is evident that they tend to blood plasma in agreement with the decrease in the capacitive behavior at high frequencies. All the results confirm and verify the physical reasonability of the equivalent circuit that has been chosen and is proposed herein.

\subsection{Single-frequency measurements}

With the aim of obtaining more accurate and informative results, measurements are generally expected to be performed using a multi-frequency approach. As previously described, the electrical impedance of blood components was measured over a

Table 1. Extracted lumped elements according to the equivalent circuit. The values of $C_{1}, \psi_{1}$ represent the common double-layer effect, while the specific intrinsic blood components behavior can be described through the values of $C_{2}, \psi_{2}$ and $R$.

\begin{tabular}{|c|c|c|c|c|c|c|}
\hline & & $\mathrm{C}_{1}(\mathrm{~F})$ & $\psi_{1}(\cdot)$ & $\mathrm{C}_{2}(\mathrm{~F})$ & $\psi_{2}(\cdot)$ & $\mathbf{R}(\mathbf{\Omega})$ \\
\hline - & red clot & $9.43 \mathrm{E}-7 \pm 7.10 \mathrm{E}-8$ & $8.53 \mathrm{E}-1 \pm 1.15 \mathrm{E}-2$ & $9.66 \mathrm{E}-11 \pm 1.40 \mathrm{E}-11$ & $8.80 \mathrm{E}-1 \pm 6.25 \mathrm{E}-2$ & $5.44 \mathrm{E}+3 \pm 3.44 \mathrm{E}+2$ \\
\hline - & whole blood concentrated & $9.21 \mathrm{E}-7 \pm 5.60 \mathrm{E}-8$ & $8.67 \mathrm{E}-1 \pm 2.31 \mathrm{E}-2$ & $4.81 \mathrm{E}-11 \pm 4.30 \mathrm{E}-12$ & $7.83 \mathrm{E}-1 \pm 5.77 \mathrm{E}-3$ & $4.46 \mathrm{E}+3 \pm 1.23 \mathrm{E}+2$ \\
\hline - & whole blood & $8.99 \mathrm{E}-7 \pm 7.70 \mathrm{E}-8$ & $8.63 \mathrm{E}-1 \pm 5.77 \mathrm{E}-3$ & $1.87 \mathrm{E}-11 \pm 6.30 \mathrm{E}-12$ & $7.40 \mathrm{E}-1 \pm 1.00 \mathrm{E}-2$ & $2.39 \mathrm{E}+3 \pm 9.14 \mathrm{E}+1$ \\
\hline- & serum high hemolysis & $8.85 \mathrm{E}-7 \pm 8.70 \mathrm{E}-8$ & $8.33 \mathrm{E}-1 \pm 5.78 \mathrm{E}-3$ & $1.02 \mathrm{E}-12 \pm 1.90 \mathrm{E}-13$ & 1 & $1.06 \mathrm{E}+3 \pm 3.76 \mathrm{E}+1$ \\
\hline-- & serum low hemolysis & $9.50 \mathrm{E}-7 \pm 6.10 \mathrm{E}-8$ & $8.37 \mathrm{E}-1 \pm 5.80 \mathrm{E}-3$ & $2.00 \mathrm{E}-12 \pm 7.20 \mathrm{E}-13$ & 1 & $8.96 \mathrm{E}+2 \pm 3.08 \mathrm{E}+1$ \\
\hline - & white clot hemolysis & $9.50 \mathrm{E}-7 \pm 1.30 \mathrm{E}-7$ & $8.13 \mathrm{E}-1 \pm 5.77 \mathrm{E}-3$ & $1.70 \mathrm{E}-11 \pm 7.10 \mathrm{E}-12$ & $7.89 \mathrm{E}-1 \pm 1.15 \mathrm{E}-3$ & $7.89 \mathrm{E}+2 \pm 1.95 \mathrm{E}+1$ \\
\hline .......... & serum & $9.70 \mathrm{E}-7 \pm 5.50 \mathrm{E}-8$ & $8.60 \mathrm{E}-1 \pm 1.00 \mathrm{E}-2$ & $4.60 \mathrm{E}-12 \pm 1.60 \mathrm{E}-12$ & 1 & $5.76 \mathrm{E}+2 \pm 1.78 \mathrm{E}+1$ \\
\hline & plasma & $9.10 \mathrm{E}-7 \pm 1.00 \mathrm{E}-7$ & $8.70 \mathrm{E}-1 \pm 1.00 \mathrm{E}-2$ & $6.40 \mathrm{E}-12 \pm 9.50 \mathrm{E}-13$ & 1 & $4.75 \mathrm{E}+2 \pm 1.01 \mathrm{E}+1$ \\
\hline .......... & white clot & $9.10 \mathrm{E}-7 \pm 6.90 \mathrm{E}-8$ & $8.90 \mathrm{E}-1 \pm 1.00 \mathrm{E}-2$ & $6.40 \mathrm{E}-12 \pm 1.40 \mathrm{E}-12$ & $9.17 \mathrm{E}-1 \pm 7.64 \mathrm{E}-2$ & $4.19 \mathrm{E}+3 \pm 1.05 \mathrm{E}+1$ \\
\hline & & $\begin{array}{r}x 10^{-7} \\
10 \\
8 \\
6 \\
4 \\
2 \\
0\end{array}$ & $\begin{array}{r}x 10^{-1} \\
8 \\
4 \\
2 \\
0\end{array}$ & $\begin{array}{rr}x 10^{-11} & \\
10 & \\
8 & \\
6 & \\
4 & \\
2 & \\
0 & \ldots \ldots \ldots\end{array}$ & $\begin{array}{r}x 10^{-1} \\
10 \\
8 \\
6 \\
4 \\
2 \\
0\end{array}$ & \begin{tabular}{r|l|l|}
$x 10^{3}$ \\
5 \\
4 \\
3 \\
2 \\
1 \\
0
\end{tabular} \\
\hline
\end{tabular}




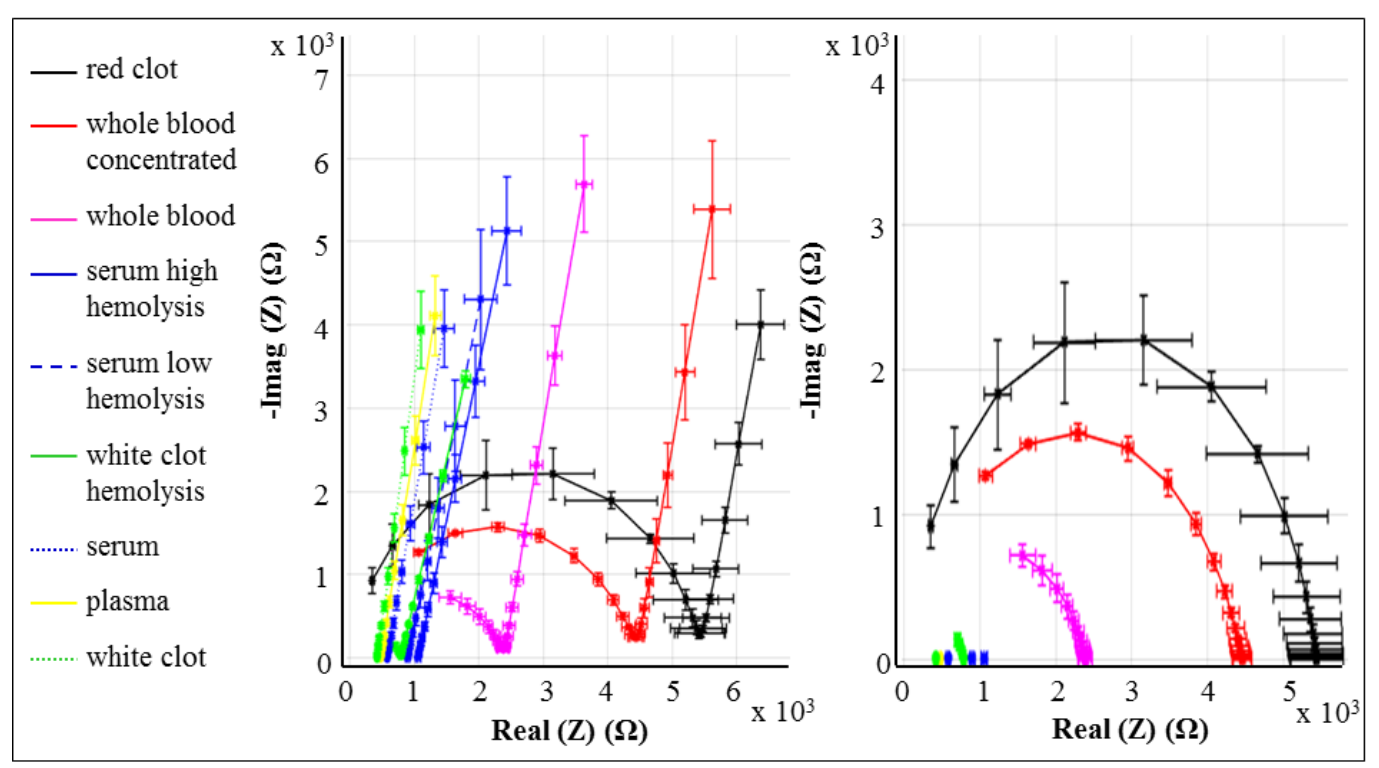

Figure 4. Electrical impedance signals displayed as Real-Imaginary plot, without (left panel) and with (right panel) the compensation of the double layer effect. Each blood component is represented in terms of mean and standard deviation, both for the Real and the Imaginary part ( $n=20$ ).

large frequency range, from $100 \mathrm{~Hz}$ to $2 \mathrm{MHz}$. Observing the signals of impedance magnitude (shown in Figure 3), we tried to identify the most ideal single frequency (among the frequencies used) in terms of accuracy and capability in discriminating the different blood components with resolution, with the purpose of improving the efficiency and performance of our measurements.

Figure 5, which follows the same color codes used in Figure 3 , shows the quantitative analysis that is used for extracting the optimal frequency in the range $[100 \mathrm{~Hz}, 2 \mathrm{MHz}$. Observing the impedance magnitude signals in Figure 3, it is clear that the best

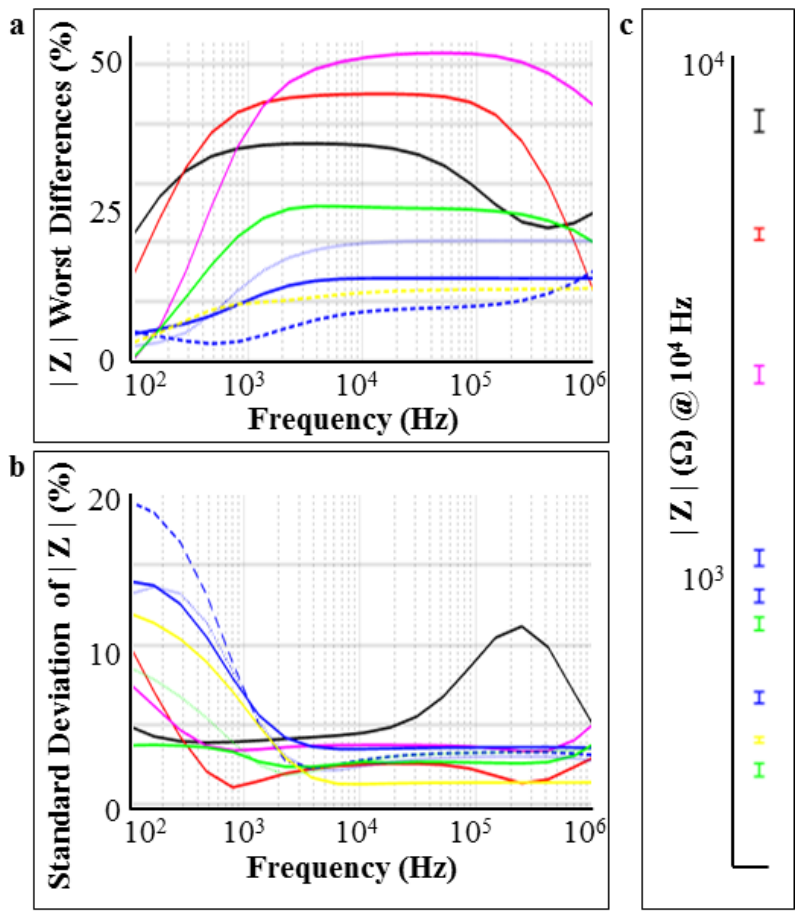

Figure 5. Optimal frequency quantification for single-frequency measurements. Worst percentage differences in impedance magnitude between adjacent blood components (a). Impedance magnitude standard deviation for each blood component, expressed as a percentage of the related average value (b). Impedance magnitude at $10^{4} \mathrm{~Hz}$, where each blood component is represented in terms of mean and standard deviation $(n=20)$. frequency that is usable for a single-frequency measurement takes place in the central interval of frequencies, where the distance between signals seems to be greater than the distance at lower or higher frequencies. According to this observation, we quantified, for all the possible pairs of signals belonging to the adjacent blood components visible in Figure 3, the difference between their percentages in terms of impedance magnitude, choosing the worst difference for representation in Figure $5 \mathrm{a}$ the minimal distance between a blood component and its adjacent one. For example, to present the black line in Figure $5 \mathrm{a}$, the percentage differences were computed between each red clot signal and each signal of whole blood concentrated, choosing, in the end, the lowest among the signals thus quantified. At the same time, we examined the trend of impedance magnitude standard deviation for each blood component, expressing it as a percentage of its average value and illustrating it in Figure $5 \mathrm{~b}$. According to the results shown in Figure $5 \mathrm{a}$ and Figure 5b, the optimal frequency is $10^{4} \mathrm{~Hz}$ because, at this frequency, all the blood components can be accurately and completely discriminated (in terms of impedance magnitude) from the adjacent ones, as well as in the worst possible case. Figure $5 \mathrm{c}$ shows the discrimination of blood components at $10^{4} \mathrm{~Hz}$.

\subsection{Impedance magnitude and hematocrit}

Hematocrit is the volume of red blood cells as a percentage of the whole blood volume, and its value can give information about the quality of blood transport and storage conditions. Hematocrit is a crucial parameter that is used to identify and quantity density variations or aggregative states in blood samples in situations of alteration that make blood unsuitable for analysis and unsuitable for transfusion. The hematocrit of each whole blood sample was measured with a commercial instrument that is commonly used in clinical laboratories and was compared with the impedance magnitude of the same sample, measured at $10^{4}$ $\mathrm{Hz}$. As shown in Figure 6, by measuring the impedance with the proposed sensor, we are able to rapidly achieve the corresponding hematocrit value. In fact, the data appears highly correlated, with a Pearson's correlation coefficient $r=0.89$, and the correlation can be linearly approximated by $y=62.9 x+6.8$, where $y$ represents the impedance magnitude and $x$ represents 


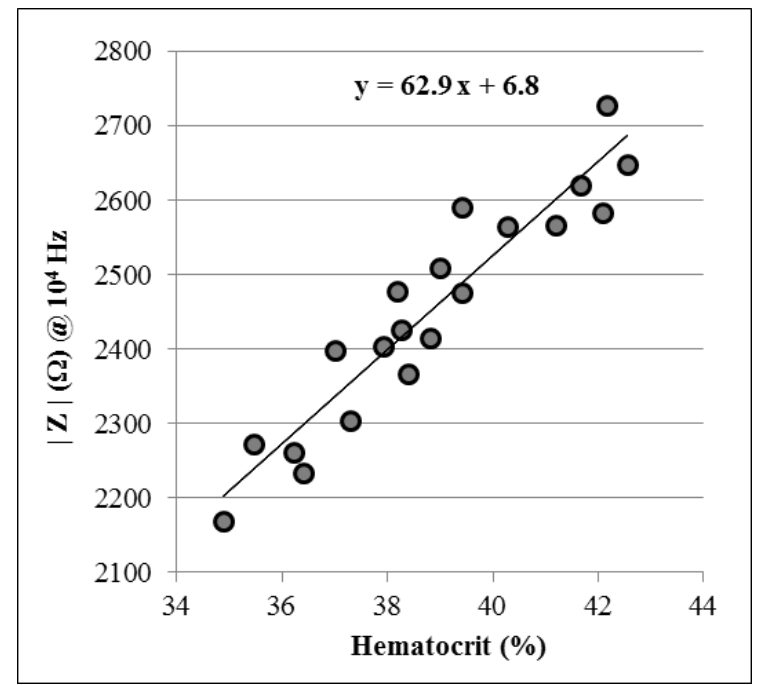

Figure 6. Quantitative comparison between the impedance magnitude at $10^{4} \mathrm{~Hz}$ and the hematocrit measured in the laboratory, for whole blood samples. The data is highly correlated (Pearson's correlation coefficient $r$ $=0.89$ ) and the correlation is linearly represented by the equation $y=62.9$ $x+6.8$, where $y$ represents the impedance magnitude and $x$ represents the hematocrit values.

the hematocrit values. The obtained results show that the proposed measuring system can be used to rapidly detect the blood hematocrit level.

\section{CONCLUSION}

Strategies in the field of risk management and patient safety are aimed at the prevention, detection, and reduction of adverse events by means of analysis of measurable and controllable errors. In clinical laboratories, the majority of critical issues occur in the pre-analytical phase, especially in activities in which the human component is still decisive. Information provided by clinical laboratories affects almost the totality of clinical decisions about diagnoses and treatments. Thus, the current focus of healthcare institutions is the improvement of patient safety, with more attention given to the prevention of pre-analytical mistakes and laboratory errors. The improvement of pre-analytical processes currently constitutes a challenge in clinical laboratories. The most frequently encountered problems are serum samples with hemolysis (in which their alteration is not visible and can compromise analytical results) and blood samples that are unsuitable for transfusion because of inappropriate sampling and storage conditions. There are different means and techniques available today, but the journey towards complete and effective rationalization of these laboratories is still long.

In this work, we presented a new simple impedance-based biosensor to perform fast impedance measurements in the preanalytical phase. Different blood components were measured and characterized in terms of impedance magnitude and phase. In particular, observing the impedance magnitude signals of serum and whole blood, we identified the upper thresholds of $700 \Omega$ and $3 \mathrm{k} \Omega$, respectively, as useful indicators for a fast check of the quality of the samples using our sensor. In addition, each blood component has been quantitatively described, estimating the parameters of the equivalent circuit proposed and finding some interesting and coherent correlations between the experimental results and the physical characteristics of the system investigated. All these results, together with the interpolation of the frequency points in the real-imaginary plot shown, have confirmed the adequacy of the equivalent circuit that has been chosen. The impedance analysis has also allowed for the quantitative identification of $10^{4} \mathrm{~Hz}$ as the optimal frequency for performing single-frequency measurements with greater efficiency and without loss of accuracy. Finally, correlation between the impedance magnitude of the whole blood at $10^{4} \mathrm{~Hz}$ and the hematocrit percentage value was achieved and quantified. Thanks to the impedance measurements and the electrical characterization of different components of blood samples, further optimization of costs, resources, and means of performing quality control (especially on serum and blood in the pre-analytical phase) has been proven as feasible. Through static electrical impedance measurements and analysis, it is possible to carry out very fast and automated controls for every sample, thus reducing the risk of errors in laboratory processes and limiting the propagation of these errors directly to the patients' health and safety.

\section{ACKNOWLEDGEMENTS}

We are grateful to Dr. A. Steffan and Dr. R. Vettori for their valuable clinical and methodological suggestions and to $\mathrm{E}$. Savaris, C. Buciol, E. Bolzonaro, and S. Bravin for their technical support.

\section{REFERENCES}

[1] G. Lippi, M. Plebani, A. M. Simundic, Quality in laboratory diagnostics: from theory to practice, Biochem. Med. 20(2) (2010) pp. 126-130.

[2] M. Plebani, Quality indicators to detect pre-analytical errors in laboratory testing, Clin. Biochem. Rev. 33(3) (2012) pp. 85-88.

[3] G. Lippi, G. C. Guidi, C. Mattiuzzi, M. Plebani, Preanalytical variability: the dark side of the moon in laboratory testing, Clin. Chem. Lab. Med. 44(4) (2006) pp. 358-365.

[4] P. Capraio, P. Plebani, Process control reduces the laboratory turnaround time, Clini. Chem. Lab. Med. 40(4) (2002) pp. 421-422.

[5] G. Lima-Oliveira, W. Volanski, G. Lippi, G. Picheth, G. C. Guidi, Pre-analytical phase management: a review of the procedures from patient preparation to laboratory analysis, Scand. J. Clin. Lab. Invest. 77(3) (2017) pp. 153-163.

[6] G. Lippi, G. L. Salvagno, N. Blanckaert, D. Giavarina, S. Green, S. Kitchen, V. Palicka, A. J. Vassault, M. Plebani, Multicenter evaluation of the hemolysis index in automated clinical chemistry systems, Clini. Chem. Lab. Med. 47(8) (2009) pp. 934-939.

[7] G. Lippi, M. Plebani, E. J. Favaloro, Technological advances in the hemostasis laboratory, Semin. Thromb. Hemost. 40(2) 2014 pp. 178-185.

[8] K. C. Gersh, C. Nagaswami, J. W. Weisel, Fibrin network structure and clot mechanical properties are altered by incorporation of erythrocytes, Thromb. Hemost. 102(6) (2009) pp. 1169-1175.

[9] P. Bhattacharjee, D. Bhattacharyya, An Insight into the Abnormal Fibrin Clots - Its Pathophysiological Roles, Fibrinolysis and Thrombolysis, Krasimir Kolev Ed., 2014, ISBN 978-953-51-12655.

[10] K. F. Lei, K. H. Chen, P. H. Tsui, N. M. Tsang, Real-time electrical impedimetric monitoring of blood coagulation process under temperature and hematocrit variations conducted in a microfluidic chip, PLoS ONE 8(10) (2013) p. e76243.

[11] A. Siegman, S. Abboud, Bioimpedance technique for monitoring cerebral artery stenosis in a 3D numerical model of the head, Med. Eng. Phys. 34(8) (2012) pp. 1095-1100.

[12] E. Barsoukov, J. R. Macdonald, Impedance Spectroscopy Theory Experiment and Applications, Wiley, New York, 2005, ISBN 978$0-471-64749-2$

[13] A. Affanni, R. Specogna, F. Trevisan, Combined electro-optical imaging for the time evolution of white thrombus growth in 
artificial capillaries, IEEE Trans. Instrum. Meas. 62(11) (2013) pp. 2954-2959.

[14] A. Affanni, R. Specogna, F. Trevisan, Ex vivo time evolution of thrombus growth through optical and electrical impedance data fusion, J. Phys. Conf. Ser. 459 (2013).

[15] A. Affanni, G. Chiorboli, L. Codecasa, M .R. Cozzi, L. De Marco, M. Mazzucato, C. Morandi, R. Specogna, M. Tartagni, F. Trevisan, A novel inversion technique for imaging thrombus volume in microchannels fusing optical and impedance data, IEEE Trans. Magn. 50(2) (2014) p. 6749262.
[16] A. Affanni, G. Chiorboli, R. Specogna, F. Trevisan, Uncertainty model of electro-optical thrombus growth estimation for early risk detection, Measurement 79 (2016) pp. 260-266.

[17] D. De Zanet, M. Battiston, E. Lombardi, R. Specogna, F. Trevisan, L. De Marco, A. Affanni, M. Mazzucato, Impedance biosensor for real-time monitoring and prediction of thrombotic individual profile in flowing blood, PLoS ONE 12 (2017) p. e0184941.

[18] Agilent Technologies, Agilent E4980A Precision LCR Meter Users Guide, 2008. 